\title{
Effect of concentration of cadmium sulfate solution on structural,optical and electric properties of Cd1-xZnxS thin films
}

\author{
Yuming Xue ${ }^{1}$, Shipeng Zhang ${ }^{1}$, Dianyou Song ${ }^{1}$, Liming Zhang ${ }^{1}$, Xinyu Wang ${ }^{1}$, Lang \\ Wang $^{1}$, and Hang Sun ${ }^{1}$ \\ ${ }^{1}$ Tianjin University of Technology
}

April 12, 2021

\begin{abstract}
Cd1-xZnxS thin films were deposited by chemical bath deposition (CBD) on glass substrate to study the influence of cadmium sulfate concentration on the structural characteristics of the thin film. The SEM results show that the thin film surfaces under the cadmium sulfate concentration of $0.005 \mathrm{M}$ exhibit better compactness and uniformity. Due to the less Cd2+ involved in the reaction and little precipitation in the solution. The distribution diagrams of thin film elements illustrate that the film growth rate changes on the increase, decrease, and increase with the increase of cadmium sulfate concentration. XRD studies exhibit the crystal structure of the film is hexagonal phase, and there are obvious diffraction peaks and better crystallinity when the concentration is $0.005 \mathrm{M}$. Spectrophotometer test results demonstrate that the relationship between zinc content $\mathrm{x}$ and optical band gap value $\mathrm{Eg}$ can be expressed by the equation $\mathrm{Eg}(\mathrm{x})=0.59 \mathrm{x} 2+0.69 \mathrm{x}+2.43$. Increasing the zinc content can increase the optical band gap, the absorbance of the thin film can be improved by decreasing the cadmium sulfate concentration, however, all of them have good transmittance. At a concentration of $0.005 \mathrm{M}$, the thin film has good absorbance in the $300-800 \mathrm{~nm}$ range, $80 \%$ transmittance, and band gap value of $3.24 \mathrm{eV}$, which is suitable for use as a buffer layer for solar cells.
\end{abstract}

\section{Hosted file}

Manuscripts.pdf available at https://authorea.com/users/407197/articles/517582-effect-ofconcentration-of-cadmium-sulfate-solution-on-structural-optical-and-electric-propertiesof-cd1-xznxs-thin-films 